\title{
2019 Update of the Korean Clinical Practice Guidelines of Stroke for Endovascular Recanalization Therapy in Patients with Acute Ischemic Stroke
}

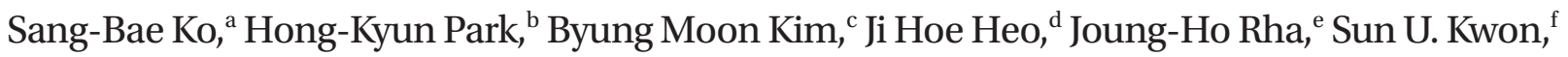

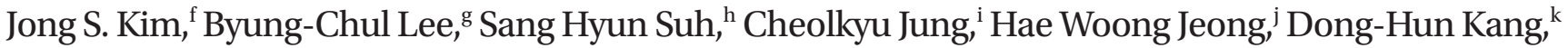 \\ Hee-Joon Bae, ${ }^{1}$ Byung-Woo Yoon, ${ }^{\mathrm{a}}$ Keun-Sik Hong ${ }^{\mathrm{b}}$ \\ aDepartment of Neurology, Seoul National University College of Medicine, Seoul, Korea \\ ${ }^{b}$ Department of Neurology, Inje University Ilsan Paik Hospital, Inje University College of Medicine, Goyang, Korea \\ 'Department of Radiology, Severance Stroke Center, Severance Hospital, Yonsei University College of Medicine, Seoul, Korea \\ ${ }^{\mathrm{d} D e p a r t m e n t}$ of Neurology, Severance Hospital, Yonsei University College of Medicine, Seoul, Korea \\ 'Department of Neurology, Inha University School of Medicine, Incheon, Korea \\ fDepartment of Neurology, Asan Medical Center, University of Ulsan College of Medicine, Seoul, Korea \\ ${ }^{9}$ Department of Neurology, Hallym University Sacred Heart Hospital, Hallym University College of Medicine, Anyang, Korea \\ hDepartment of Radiology, Gangnam Severance Hospital, Yonsei University College of Medicine, Seoul, Korea \\ 'Department of Radiology, Seoul National University Bundang Hospital, Seoul National University College of Medicine, Seongnam, Korea \\ 'Department of Radiology, Inje University Busan Paik Hospital, Inje University College of Medicine, Busan, Korea \\ ${ }^{k}$ Department of Neurosurgery, School of Medicine, Kyungpook National University, Daegu, Korea \\ 'Department of Neurology and Cerebrovascular Center, Seoul National University Bundang Hospital, Seoul National University College of Medicine, \\ Seongnam, Korea
}

Endovascular recanalization therapy (ERT) has been a standard of care for patients with acute ischemic stroke due to large artery occlusion (LAO) within 6 hours after onset, since five landmark ERT trials conducted by 2015 demonstrated its clinical benefit. Recently, two randomized clinical trials demonstrated that ERT, even in the late time window of up to 16 hours or 24 hours after last known normal time, improved the outcome of patients who had a target mismatch, defined as either clinical-core mismatch or perfusion-core mismatch, which prompted the update of national guidelines in several countries. Accordingly, to provide evidence-based and up-to-date recommendations for ERT in patients with acute LAO in Korea, the Clinical Practice Guidelines Committee of the Korean Stroke Society decided to revise the previous Korean Clinical Practice Guidelines of Stroke for ERT. For this update, the members of the writing group were appointed by the Korean Stroke Society and the Korean Society of Interventional Neuroradiology. After thoroughly reviewing the updated evidence from two recent trials and relevant literature, the writing members revised recommendations, for which formal consensus was achieved by convening an expert panel composed of 45 experts from the participating academic societies. The current guidelines are intended to help healthcare providers, patients, and their caregivers make well-informed decisions and to improve the quality of care regarding ERT. The ultimate decision for ERT in a particular patient must be made in light of circumstances specific to that patient.
Correspondence: Keun-Sik Hong Department of Neurology, Inje University Ilsan Paik Hospital, Inje University College of Medicine, 170 Juhwa-ro, llsanseo-gu, Goyang 10380, Korea

Tel: +82-31-910-7680

Fax: $+82-31-910-7368$

E-mail: nrhks@paik.ac.kr

Received: January 3, 2019 Revised: January 16, 2019 Accepted: February 18, 2019

Keywords Guideline; Cerebral infarction; Large cerebral artery occlusion; Reperfusion; Mechanical thrombolysis 


\section{Introduction}

Previously, five pivotal randomized clinical trials (RCTs) and their meta-analysis clearly demonstrated the benefit of endovascular recanalization therapy (ERT), primarily with stent retrievers, in patients with acute anterior circulation ischemic stroke due to large artery occlusion (LAO) ${ }^{1-6}$ Of the five RCTs, Multicenter Randomized Clinical trial of Endovascular treatment for Acute ischemic stroke in the Netherlands (MR CLEAN), Extending the Time for Thrombolysis in Emergency Neurological Deficits-Intra-Arterial (EXTEND-IA), and Solitaire with the Intention for Thrombectomy as Primary Endovascular Treatment (SWIFT PRIME) enrolled patients within 6 hours from stroke onset. ${ }^{1-3}$ Revascularization with Solitaire FR Device versus Best Medical Therapy in the Treatment of Acute Stroke Due to Anterior Circulation Large Vessel Occlusion Presenting within Eight Hours of Symptom Onset (REVASCAT) and Endovascular Treatment for Small Core and Proximal Occlusion Ischemic Stroke (ESCAPE) enrolled patients up to 8 or 12 hours from stroke onset, but $90.3 \%$ of patients in REVASCAT and 84.5\% of those in ESCAPE were enrolled within 6 hours. ${ }^{4,5}$ Therefore, major guidelines and statements endorsed ERT up to 6 hours after symptom onset in patients with $L A O$ in the anterior circulation with the highest level of evidence (LOE) and the strongest grade of recommendation (GOR). ${ }^{7-10}$ According to the guidelines, the benefit of ERT was uncertain after 6 hours of symptom onset. ${ }^{7.10}$ Specifically, the previous Korean guidelines recommended that "ERT can be considered for patients having favorable multimodal imaging profiles regarding expected benefit and safety (LOE IV, GOR C)."10

The proposition "time is brain" also applies to ERT as well as to intravenous thrombolysis. The Highly Effective Reperfusion evaluated in Multiple Endovascular Stroke Trials (HERMES) collaborators showed that the benefit of ERT declined with increasing time from stroke onset to the initiation of ERT, and the benefit was not significant after 7.3 hours. ${ }^{.1}$ However, observational studies have claimed that, even in the late time window, many patients still have salvageable tissue readily identified with advanced stroke imaging and could benefit from reperfusion therapy. ${ }^{12-15}$ Based on these concepts, diffusion-weighted imaging (DWI) or computerized tomography perfusion (CTP) assessment with Clinical Mismatch in the Triage of Wake Up and Late Presenting Strokes Undergoing Neurointervention with Trevo (DAWN) and Endovascular Therapy Following Imaging Evaluation for Ischemic Stroke 3 (DEFUSE 3) were designed. ${ }^{16,17}$ These confirmed the benefit of ERT in patients who had target mismatch, assessed by either clinicalcore mismatch or with perfusion-core mismatch in the ex- tended time window up to 16 or 24 hours after last known normal time (LNT). ${ }^{18,19}$ The DAWN and DEFUSE 3 results prompted the revision of guidelines of United States, Canada, and Australia. ${ }^{20-22}$ The Clinical Practice Guideline (CPG) Committee of the Korean Stroke Society also decided to update the Korean CPG for Stroke, to provide evidence-based and up-todate recommendations for ERT in patients with acute LAO. The purpose of the current guidelines is to help guide well-informed decisions and to improve the quality of care regarding ERT. The ultimate decision for ERT in individual patients must be made by the responsible healthcare providers and patients and/or their caregivers in light of circumstances specific to the individual patient.

\section{Previous guidelines}

The previous 2016 Korean CPG for ERT in patients with acute ischemic stroke provided the following recommendations, which were generated by the available evidence up to May 2015. ${ }^{10}$

\section{Endovascular recanalization therapy}

1. In patients with major ischemic stroke due to an acute LAO in the anterior circulation (internal carotid artery, $\mathrm{M} 1$, and possibly large M2 branch) within 6 hours, ERT is recommended to improve clinical outcomes (LOE la, GOR A).

2. In patients eligible for intravenous tissue plasminogen activator (IV-TPA), administration of IV-TPA is recommended before the initiation of ERT (LOE la, GOR A). Since IV-TPA should not significantly delay ERT, it is recommended to simultaneously proceed ERT during IV-TPA treatment without waiting for clinical response to IV-TPA.

3. In patients who are contraindicated for IV-TPA, ERT is recommended as a first-line therapy in patients with major ischemic stroke due to an acute LAO in the anterior circulation within 6 hours (LOE Ila, GOR B).

4. In patients with major ischemic stroke due to acute LAO in the poster circulation (basilar artery, $\mathrm{P} 1$, and vertebral artery) within 6 hours, ERT can be considered (LOE III, GOR B).

5. For patients with acute $L A O$ in the anterior or posterior circulation presenting after 6 hours, ERT can be considered for patients having favorable multimodal imaging profiles regarding expected benefit and safety. Each center is encouraged to define own selection criteria (LOE IV, GOR C).

6. If indicated, ERT should be initiated as fast as possible (LOE Ila, GOR B).

7. Stent-retriever thrombectomy is recommended as a first-line ERT (LOE la, GOR A).

8. If recanalization is not achieved with stent-retriever thrombec- 
tomy, the addition of other ERT modalities can be considered after taking into account the expected efficacy and safety (LOE IV, GOR C).

9. Other mechanical thrombectomy or thrombus aspiration devices may be considered as a first-line modality at the discretion of responsible interventionists after taking into account technical aspects (LOE IV, GOR C).

10. During ERT, conscious sedation is generally preferred to general anesthesia. However, the decision should be made after consideration of patient's condition and center's experience (LOE III, GOR B).

\section{Neuroimaging evaluation}

1. Non-contrast computed tomography (CT) or magnetic resonance imaging (MRI) should be conducted to exclude hemorrhagic stroke or other non-stroke etiologies (good practice points [GPPs]).

2. Non-invasive vascular imaging (CT angiography or magnetic resonance [MR] angiography) is recommended to confirm acute LAO for patients with major ischemic stroke (GPP).

3. For patients who are not able to perform non-invasive vascular imaging, stroke severity or clot sign on non-contrast CT can guide decision for ERT (GPP).

4. For selecting patients, neuroimaging evaluation for extensive early ischemic injury can guide decision for ERT (GPP).

5. Advanced multimodal imaging to assess collaterals, extent of ischemic core, or perfusion-diffusion mismatch can be considered to identify patients who are likely to benefit from ERT (GPP). However, the multimodal imaging should not significantly delay ERT.

\section{System organization}

1. For centers capable of providing ERT, the organization and implementation of critical pathway and formal protocol are recommended to accelerate the delivery of ERT (GPP).

2. For centers that are not adequately staffed for ERT, it is encouraged to have a referral plan to a center capable of ERT for patients eligible for ERT. If indicated, initiating IV-TPA before referral is encouraged (GPP).

3. Each center is encouraged to define own criteria for the multidisciplinary ERT team that is responsible for initial evaluation, decision making, and ERT procedure (GPP).

4. To assess and improve the quality of ERT, each center is encouraged to monitor key time metrics of door-to-neuroimaging and door-to-groin puncture (GPP).

5. It is encouraged to assess functional outcome, recanalization rate, and complication rate after ERT (GPP).

\section{Methodology}

\section{Process of the update of the Korean CPG for ERT}

In May 2018, the Guideline Oversight Committee of the Korean Stroke Society, taking into account the new evidence of ERT benefit in the extended time window and its applicability to clinical practice in Korea, approved the processes of updating the Korean CPG for ERT with regard to purpose and scope, and appointed an expert panel for consensus achievement of the recommendations prepared by the writing committee members.

The CPG Committee of the Korean Stroke Society assembled the writing members appointed by the Korean Stroke Society and the Korean Society of Interventional Neuroradiology. In addition, we organized an expert panel consisting of 45 experts (30 from the Korean Stroke Society and 15 from the Korean Society of Interventional Neuroradiology) to achieve consensus for the recommendations proposed by the writing group members.

In September 2018, the expert panel members reached a consensus following a modified Delphi method and the Guideline Oversight Committee and the participating academic societies reviewed and approved the draft prepared by the writing members.

\section{Evidence search and data analysis}

To review the updated information on ERT in the extended time window since the publication of the previous CPG in 2016, we reviewed and assessed RCT results, updated national guidelines (published or available online), and relevant articles solicited from experts.

\section{LOE and GORs}

We determined the LOE and the GOR for each recommendation based on the suggestion of the US Agency for Healthcare Policy and Research (currently the Agency for Healthcare Research and Quality) (Table 1). ${ }^{23}$ We used this grading system to accord with previous Korean CPGs for stroke.

\section{Evidence summary}

\section{ERT benefit in the extended time window of 6 to 24 hours from stroke onset}

\section{DAWN}

DAWN selected patients with LAO in the anterior circulation who could be randomized between 6 and 24 hours from LNT and who had a target mismatch between clinical deficit and ischemic core. ${ }^{18}$ From a total of 206 patients enrolled (mean age, 70.0 years; female, 54.9\%), 107 were randomized to the ERT 
Table 1. Level of evidence and grade of recommendation

\begin{tabular}{ll}
\hline LOE & \\
la & Evidence obtained from meta-analysis of randomized controlled trials \\
Ib & Evidence obtained from at least one randomized controlled trial \\
Ila & Evidence obtained from at least one well-designed controlled study without randomization \\
IIb & Evidence obtained from at least one other type of well-designed quasi-experimental study \\
III & Evidence obtained from well-designed non-experimental descriptive studies, such as comparative studies, correlation studies and case \\
IV & Evidies \\
GOR & Required: at least one randomized controlled trial as part of the body of literature of overall good quality and consistency addressing \\
A (LOE la, Ib) & Required: availability of well conducted clinical studies but no randomized clinical trials on the topic of recommendation \\
B (LOE Ila, IIb, III) & Required: evidence obtained from expert committee reports or opinions and/or clinical experiences of respected authorities. This grade \\
C (LOE IV) & indicates absence of directly applicable clinical studies of good quality \\
GPP & Recommended best practice based on the clinical experience of the guideline development group
\end{tabular}

$L O E$, level of evidence; $G O R$, grade of recommendation; GPP, good practice point.

group (105 patients underwent ERT) and 99 to the control group. The majority of the patients (78\%) had an occlusion in the first segment of the middle cerebral artery (MCA), followed by the intracranial internal carotid artery (19.9\%) and the second segment of MCA (2.4\%). The median interval from LNT to randomization was 12.2 hours versus 13.3 hours, the baseline median National Institute of Health Stroke Scale (NIHSS) score was 17 versus 17, and the median infarct volume at baseline was 7.6 and $8.9 \mathrm{~mL}$ in the ERT group and the control group, respectively.

The primary endpoint, the mean score for disability on the 90-day utility-weighted modified Rankin Scale (mRS) (range, 0 [death] to 10 [no symptoms or disability]), was significantly higher in the ERT group than in the control group (5.5 points vs. 3.4 points; adjusted difference, 2.0 points; $95 \%$ confidence interval $[\mathrm{Cl}], 1.1$ to 3.0 ; posterior probability of superiority, $>0.999$ ). In a conventional dichotomized mRS analysis, the proportion of 90-day mRS 0-2 was also significantly higher in the ERT group than in the control group (49\% vs. $13 \%$; adjusted difference, 33\%; 95\% credible interval, 21 to 44; posterior probability of superiority, $>0.999$ ). There was no heterogeneity of the treatment effect on the utility-weighted mRS score in any of the pre-specified subgroups. The findings indicate that, for every two patients treated with ERT, one additional patient had a clinically meaningful improvement in the 90-day disability degree, and for every 2.8 patients treated with ERT, one additional patient had a 90-day functional independence (mRS $0-2)$. There were no differences in the rate of symptomatic intracranial hemorrhage ( $6 \%$ vs. $3 \%, P=0.50$ ) and 90 -day mortality (19\% vs. $18 \%, P=1.00$ ) between the ERT and control groups.

The clinical benefit observed in DAWN was driven by suc- cessful reperfusion with ERT. In the ERT arm, 84\% of patients achieved successful recanalization defined as grade $2 b$ or 3 on the modified Thrombolysis in Cerebral Infarction (mTICl) scale at a median interval of 13.6 hours from LNT to reperfusion. The ERT group compared to the control group had significantly higher rates of early response (decrease in the NIHSS score $\geq 10$ points or an NIHSS score 0-1 within 7 days or at discharge) and recanalization at 24 hours, and had significantly lower infarct volume and infarct growth at 24 hours.

\section{DEFUSE 3}

DEFUSE 3 selected patients with LAO in the anterior circulation who could be started on ERT in 6 to 16 hours from LNT and who had a target mismatch assessed by ischemic core and penumbral regions from CTP or diffusion and perfusion MRI images. ${ }^{19}$ Of the 182 patients enrolled (median age, 70 years; female, 50.5\%), 92 were randomized to the ERT group (90 patients underwent ERT) and 90 to the control group. In 114 patients, the occlusion site was the MCA (63\%; 113 in the M1 segment and one in the M2 segment) and in 68 (37\%), the intracranial ICA. In the ERT group versus the control group, the median interval from LNT to randomization was 10.9 hours versus 10.7 hours; the baseline median NIHSS score, 16 versus 16; the median ischemic core volume at baseline, $9.4 \mathrm{~mL}$ versus $10.1 \mathrm{~mL}$; and the median perfusion deficit volume, 114.7 $\mathrm{mL}$ versus $116.1 \mathrm{~mL}$; respectively.

The primary endpoint, the 90-day mRS score distribution, was more favorable in the ERT group than in the control group (unadjusted common odds ratio, $2.77 ; 95 \% \mathrm{Cl}_{1} 1.63$ to 4.70 ; $P<0.001)$. The proportion of patients who achieved 90 -day $\mathrm{mRS}$ 
0-2 was substantially higher in the ERT group than in the control group (45\% vs. 17\%; unadjusted risk ratio, $2.67 ; 95 \% \mathrm{Cl}$, 1.60 to $4.48 ; P<0.001)$. The treatment effect on the 90 -day mRS distribution was consistent across the pre-specified subgroups.

In the ERT group, 76\% achieved mTICl $2 b$ or 3 reperfusion at median interval of 11.5 hours from LNT. At 24 hours, infarct volume ( $35 \mathrm{~mL}$ vs. $41 \mathrm{~mL}$ ) and infarct growth ( $23 \mathrm{~mL}$ vs. $33 \mathrm{~mL}$ ) were lower in the ERT group than in the control group, but the difference was not statistically significant. However, the rates of 24-hour complete recanalization and reperfusion $>90 \%$ were increased (over 4-fold) with ERT.

There was no difference in the rate of symptomatic intracranial hemorrhage between the ERT group and the control group ( $7 \%$ vs. $4 \%, P=0.75$ ), but the 90 -day mortality rate was marginally lower in the ERT group than in the control group (14\% vs. $26 \%, P=0.05$ ).

\section{Patient selection for ERT in the extended time window}

\section{Time window}

The time window differed between DAWN and DEFUSE 3; the eligible time window from LNT was 6 to 24 hours in DAWN and 6 to 16 hours in DEFUSE 3. However, the median interval between LNT and randomization was 12.8 hours in DAWN and 10.8 hours in DEFUSE 3 , and the median interval between LNT and reperfusion in the ERT group was 13.6 hours in DAWN and 12.1 hours in DEFUSE 3. Therefore, patients enrolled in the two trials had a similar time window of about 12 hours from LNT and a comparable interval from LNT to reperfusion of about 13 hours for patients receiving ERT.

In subgroup analyses, the ERT benefit remained significant in patients randomized after 12 hours in both trials. In DEFUSE 3 , there was a trend of greater ERT benefit in patients randomized after 12 hours, although the interaction of the treatment effect to the time of randomization was not significant. Taken together, the common time window of the two trials was up to 16 hours, but ERT may benefit up to 24 hours in highly selected patients.

\section{Identification of patients with target mismatch in DAWN and DEFUSE 3}

DAWN used clinical-core mismatch that was adjusted by age and stroke severity. ${ }^{16,18}$ For patients who were $\geq 80$ years and had the NIHSS score $\geq 10$, the ischemic core volume should be less than $21 \mathrm{~mL}$. For patients aged $<80$ years, the ischemic core volume should be less than $31 \mathrm{~mL}$ in those with the NIHSS score 10 to 19 , and it should be $31 \mathrm{~mL}$ to less than $51 \mathrm{~mL}$ in those with the NIHSS score $\geq 20$. The ischemic core volume was assessed by DWI or CTP using the automated software (RAPID,
iSchemaView, Menlo Park, CA, USA).

DEFUSE 3 used perfusion-core mismatch and the eligible mismatch criteria from CTP or DWI/perfusion-weighted imaging (PWI) MRI scans were (1) an ischemic core volume $<70 \mathrm{~mL}$; (2) a ratio of ischemic tissue volume (perfusion deficit area defined with time to maximum of residue function $\left[T_{\max }\right.$ ] exceeding 6 seconds on perfusion imaging) to initial ischemic core volume $\geq 1.8$; and (3) an absolute penumbra volume $\geq 15 \mathrm{~mL} .{ }^{19}$ DEFUSE 3 used the automated software (RAPID) to assess the imaging profiles. Moreover, DEFUSE 3 allowed enrollments of patients with LAO in the anterior circulation and small ischemic core (DWI volume $<25 \mathrm{~mL}$ ) if PWI MRI or CTP was technically inadequate.

The two trials differed in the evaluation of target mismatch: clinical-core versus perfusion-core mismatch. In addition, to assess ischemic core volume, the two trials used the same automated program (RAPID), although the thresholds of ischemic core volume for eligibility were different. However, patients enrolled in DAWN and DEFUSE 3 were generally comparable; for patients treated with ERT, the median ischemic core volume was $7.6 \mathrm{~mL}$ (interquartile range [IQR], 2.0 to 18.0 ) in DAWN and $9.4 \mathrm{~mL}(I \mathrm{R}, 2.3$ to 25.6) in DEFUSE 3, and the median NIHSS score was $17 \mathrm{~mL}$ (IQR, 13 to 21) in DAWN and $16 \mathrm{~mL}$ (IQR, 10 to 20) in DEFUSE 3. The control groups in the two trials also had comparable characteristics. Therefore, small ischemic core and severe deficit-whether it was defined by clinical measure or perfusion imaging-attributed to ICA or M1 segment, were the key features of the enrolled patients.

\section{Identifying patients with target mismatch in real world clinical practice}

The assessment of ischemic core or target mismatch in patients presenting beyond 6 hours with the automated software that was used and qualified by the DAWN and DEFUSE 3 trials was preferred. The 2018 United States guidelines recommend adhering to the DAWN or DEFUSE 3 eligibility criteria. ${ }^{20}$ The 2018 updated Canadian stroke guidelines also recommend following the DAWN and DEFUSE 3 criteria. Given that a CT-based approach rather than an MRI-based approach is more available in most Canadian centers, the Canadian guidelines recommend CTP imaging and the use of software that can provide reproducible objective measurements of ischemic core and penumbra. ${ }^{21}$ In contrast, the Australian guidelines provide a more flexible recommendation to undertake ERT between 6 to 24 hours from LNT if clinical and CTP or MRI features indicate the presence of salvageable brain tissue. ${ }^{22}$ In Korea, most stroke centers are not equipped with the RAPID software, and accordingly they need to define their own imaging and/or clinical criteria that can be readily available on-site and reasonably select eligible patients. 
It may be an issue how reliably stroke experts can identify patients eligible for ERT based on clinical features and multimodal imaging readily available on-site, without relying on a validated software program. Previously, the DEFUSE 3 investigators conducted a multicenter cohort study, which aimed to evaluate the validity of CTP assessed by the RAPID software for identifying target mismatch in patients treated with ERT as a foundation study for DEFUSE 3. For the decision making for ERT, the participating investigators used non-contrast CT, CT angiogram (CTA), and CTP of their own routine protocols, and were instructed not to use the RAPID software. ${ }^{13}$ Among the total of 190 patients treated with ERT based on their clinical and imaging features, 131 patients (69\%) were later identified to have target mismatch on the RAPID software assessment. Of note, the proportion of patients having target mismatch was $62 \%$ in the $<6$-hour window, but increased to $80 \%$ in the $>6$ hour window (83\% in the 6- to 9-hour window, 85\% in the 9to 12-hour window, and 69\% in the $>12$-hour window). Among 170 patients who achieved successful reperfusion, the proportion of 90-day mRS 0-2 was 59\% $(n=100)$, which was less than $66 \%$ among patients $(n=114)$ who had mismatch profile and achieved reperfusion, but seemed acceptably high. Furthermore, among all 190 patients, the proportion of 90-day mRS 0-2 was about 55\%, which is likely higher than the 46\% observed in the ERT arm of the HERMES collaborative study. The rate of symptomatic intracerebral hemorrhage was 5.2\% of all patients and was not influenced by the target mismatch status. Taken together, even without automated software processing, stroke experts appeared to identify reasonably patients who had target mismatch with clinical features and routinely available multimodal imaging, even more selectively identifying patients presenting in the later time window.

Academic and commercial automated programs have been developed and are under development. It is generally accepted that, for the assessment of ischemic core and/or target mismatch, automated quantitative imaging analyses outperform qualitative measures, and some centers in Korea might currently use one of these programs in clinical practice. However, comparative studies showed that there were discrepancies in perfusion maps generated among commercial programs, and academic programs compared to commercial programs were generally more accurate in the analysis of perfusion maps. ${ }^{24-26}$ Therefore, clinicians should recognize the limitations of each software program available in their centers.

Several studies have clearly demonstrated that patients with good collateral circulation had a greater benefit from ERT. DAWN and DEFUSE 3 did not consider the status of collateral circulation for eligibility and the effect of collateral circulation status on the ERT benefit has not been well studied in the late time window. In general, patients with clear onset and a small ischemic core, even in the late time window, are likely to have good collateral circulation. However, in patients with unknown onset time, the small ischemic core does not always indicate good collateral circulation, which could determine the speed of infarct progression and the response to ERT. The international stroke expert groups strongly recommend to assess collateral circulation assessment as well as LAO, ischemic core, and target mismatch for potential ERT candidates. ${ }^{27}$ The Canadian guidelines also suggest that moderate-to-good collateral status on CTA predict a better response to ERT. ${ }^{21}$ Because of lack of clear evidence in the extended time window, no specific recommendation for the collateral assessment is provided in the current updated guidelines, but it should be considered for patient selection, especially for those with unclear onset time.

\section{Consensus achievement}

The writing members prepared the updated recommendations based on available evidence and their practical applicability to the healthcare system in Korea. To achieve consensus for the proposed recommendations using a modified Delphi method, we convened a panel of 45 experts of neurologists, interventional radiologists, and neurosurgeons: 30 from the Korean Stroke Society and 15 from the Korean Society of Interventional Neuroradiology. Using a 9-point scale (a score of 9 as strong agreement and a score of 1 as strong disagreement) modified from the RAND Corporation method, ${ }^{28}$ the expert panel members were asked to individually provide their ratings on each recommendation. We defined scores 7-9 as agreement, 4-6 as uncertainty, and 1-3 as disagreement. For each recommendation, if $\geq 75 \%$ of the participating panel members agreed (providing a 7-9 score), it was regarded as a consensus for that recommendation. If the agreement rate was $<75 \%$, additional Delphi rounds were planned with revised recommendations.

The current updated guidelines are intended to focus on the ERT in the extended time window. Therefore, the panel members were first asked whether it is necessary to update the recommendation of ERT between 6 to 24 hours from LNT. They were also asked whether multimodal imaging available on-site other than the RAPID software program could be used to select patients eligible for ERT between 6 to 24 hours. Then, they were asked to choose one of the following recommendations, taking into account evidence and applicability in Korea. (1) "In selected patients with acute ischemic stroke due to LAO in the anterior circulation presenting within 6 to 24 hours from last seen normal, ERT is recommended when the patients meet the 
Table 2. Summary of current recommendations

Comment

ERT

1. In patients with major ischemic stroke due to an acute large artery occlusion in the anterior circulation (internal carotid artery, M1, and possibly large M2 branch) within 6 hours, ERT is recommended to improve clinical outcomes (LOE la, GOR A).

2. In patients eligible for IV-TPA, administration of IV-TPA is recommended before the initiation of ERT (LOE Ia, GOR A). Since No change IV-TPA should not significantly delay ERT, it is recommended to simultaneously proceed ERT during IV-TPA treatment without waiting for clinical response to IV-TPA.

3. In patients who are contraindicated for IV-TPA, ERT is recommended as a first-line therapy in patients with major ischemic No change stroke due to an acute large artery occlusion in the anterior circulation within 6 hours (LOE Ila, GOR B).

4. In patients with major ischemic stroke due to acute large artery occlusion in the poster circulation (basilar artery, P1, and No change vertebral artery) within 6 hours, ERT can be considered (LOE III, GOR B).

5. In selected patients with acute ischemic stroke due to large vessel occlusion in the anterior circulation presenting within 6 to 24 hours from last seen normal, ERT can be recommended when the patients have target mismatch assessed by multimodal imaging and/or clinical deficit \& when reperfusion by ERT is expected to improve the outcome of patients. For patient selection, each institution is recommended to have its own criteria, which can help in the timely and reasonable identification of patients with target mismatch in the late time window (LOE III, GOR C).

6. In selected patients with acute ischemic stroke due to large vessel occlusion in the posterior circulation presenting after 6 hours, ERT can be considered for patients having favorable multimodal imaging profiles in consideration of risks and benefits. Each center is encouraged to define its own patient selection criteria (LOE IV, GOR C).

If indicated, ERT should be initiated as fast as possible (LOE Ila, GOR B).

7. Stent-retriever thrombectomy is recommended as a first-line ERT (LOE la, GOR A).

8. If recanalization is not achieved with stent-retriever thrombectomy, the addition of other ERT modalities can be considered after taking into account the expected efficacy and safety (LOE IV, GOR C).

9. Other mechanical thrombectomy or thrombus aspiration devices may be considered as a first-line modality at the discretion of responsible interventionists after taking into account technical aspects (LOE IV, GOR C).

10. During ERT, conscious sedation is generally preferred to general anesthesia. However, the decision should be made after consideration of patient's condition and center's experience (LOE III, GOR B).

Neuroimaging evaluation

1. Non-contrast CT or MRI should be conducted to exclude hemorrhagic stroke or other non-stroke etiologies (LOE III, GOR C).

2. Non-invasive vascular imaging (CT angiography or MR angiography) is recommended to confirm acute large artery occlusion for patients with major ischemic stroke (LOE III, GOR C).

3. For patients who are not able to perform non-invasive vascular imaging, stroke severity or clot sign on non-contrast CT can guide decision for ERT (GPP).

4. For selecting patients, neuroimaging evaluation for extensive early ischemic injury can guide decision for ERT (GPP).

5. In selected patients who present within 6 to 24 hours from last seen normal, multimodal imaging for assessing collaterals, infarct core, or perfusion (or clinical)-diffusion mismatch is recommended to select eligible patients for ERT. Each center is encouraged to define its own imaging modality to timely identify target mismatch (LOE III, GOR C).

System organization

1. For centers capable of providing ERT, the organization and implementation of a critical pathway and a formal protocol are No change recommended to accelerate the delivery of ERT (GPP). 2. For centers that are not adequately staffed for ERT, it is encouraged to have a referral plan to a center capable of ERT for No change
patients eligible for ERT. If indicated, initiating IV-TPA before referral is encouraged (GPP).

3. Each center is encouraged to define own criteria for the multidisciplinary ERT team that is responsible for initial evalua- No change tion, decision-making, and ERT procedure (GPP).

4. To assess and improve the quality of ERT, each center is encouraged to monitor key time metrics of door-to-neuroimaging No change and door-to-groin puncture (GPP).

5. It is encouraged to assess functional outcome, recanalization rate, and complication rate after ERT (GPP).

No change

ERT, endovascular recanalization therapy; LOE, level of evidence; GOR, grade of recommendation; IV-TPA, intravenous tissue plasminogen activator; CT, computed tomography; MRI, magnetic resonance imaging; MR, magnetic resonance; GPP, good practice point. 
DAWN or DEFUSE 3 eligibility criteria (LOE Ib, GOR A)" or (2) "In selected patients with acute ischemic stroke due to LAO in the anterior circulation presenting within 6 to 24 hours from last seen normal, ERT can be recommended when the patients have target mismatch assessed by multimodal imaging and/or clinical deficit \& when reperfusion by ERT is expected to improve the outcome of the patients. For patient selection, each institution is recommended to have its own criteria, which can timely and reasonably identify patients with target mismatch in the late time window (LOE III, GOR C)." For the second proposal, we graded the LOE as III and the GOR as C because the validity of multimodal imaging available on-site has not been confirmed by clinical trials.

Urgent neuroimaging studies are necessary to exclude hemorrhagic stroke or stroke mimics. In a cost-effective analysis, immediate CT scanning improved clinical outcome partly attributed to timely management decision and appropriate treatment, which translated to better quality of life. ${ }^{29}$ In addition, MRI versus CT was comparable to detect hemorrhagic stroke or acute vascular lesion. ${ }^{30,31}$ In addition, for potential ERT candidates, an LAO should be confirmed by non-invasive vascular imaging (CTA or MR angiography). Since these pieces of evidence were not reflected in the previous recommendation, the panel members were asked whether they agreed to upgrade the LOE and GOR from 'GPP' to 'LOE III and GOR C' for the recommendations of non-contrast CT or MRI to exclude hemorrhagic stroke or other non-stroke etiologies and non-invasive vascular imaging for patients with major ischemic stroke.

Among the 45 experts invited, 41 (91\%) provided their ratings (list of responding participants in Supplementary Table 1). In the first Delphi round, consensus was achieved in all question items and recommendations, and the scores and agreement rates were generally high (Supplementary Table 2). In particular, for the selection of patients eligible for ERT in the extended time window, the expert panel members agreed to recommend multimodal imaging available on-site rather than the specific RAPID software program. The final draft of the current guidelines was reviewed and approved by the participating academic societies.

\section{Recommendations}

Updated Recommendations for ERT, neuroimaging evaluation, and system organization are summarized in Table 2 and Supplementary Table 3.

\section{Impact of expanding the time window of ERT on clinical practice in Korea}

Expanding the time window of ERT up to 24 hours will have a significant impact on the stroke care system in Korea. In a single comprehensive center study in United States, only 1.7\% of all patients with acute ischemic stroke admitted met the DAWN trial eligibility and $2.7 \%$ met the DAWN or DEFSUE-3 criteria. $^{32}$ In a Korean study analyzing 6,742 patients enrolled in a multicenter registry between May 2011 and December 2012, 1.1\% met the DAWN criteria. Based on these observations, the ageand sex-specific ischemic stroke incidence data ${ }_{1}{ }^{33}$ and the 2016 Korean population data, the estimated nationwide annual number of patients who could meet the DAWN criteria was 1,331 in Korea. ${ }^{34}$ Therefore, despite the small number of patients eligible for the extended ERT time window in individual centers, the nationwide number of patients would not be negligible. According to the 2017 nationwide insurance claim database, approximately 3,500 patients were treated with ERT in Korea (personal communication with Byung Moon Kim in 2018). Therefore, ERT candidates could be increased by $38 \%$ by expanding the time window for ERT. However, to identify these patients, an additional $23.7 \%$ of all patients with acute ischemic stroke should be urgently screened. ${ }^{34}$ Furthermore, given that patients with suspected stroke should also be screened in the extended time window, responsible stroke experts will have a substantially increased burden, and should be supported accordingly with corresponding resource allocation.

\section{Supplementary materials}

Supplementary materials related to this article can be found online at https://doi.org/10.5853/jos.2019.00024.

\section{Disclosure}

The authors have no financial conflicts of interest.

\section{References}

1. Berkhemer $O A$, Fransen PS, Beumer $D$, van den Berg LA, Lingsma HF, Yoo Av, et al. A randomized trial of intraarterial treatment for acute ischemic stroke. N Engl J Med 2015;372:11-20.

2. Campbell BC, Mitchell PJ, Kleinig TJ, Dewey HM, Churilov L, Yassi $N$, et al. Endovascular therapy for ischemic stroke with perfusion-imaging selection. N Engl J Med 2015;372:10091018.

3. Saver JL, Goyal M, Bonafe A, Diener HC, Levy El, Pereira VM, 
et al. Stent-retriever thrombectomy after intravenous t-PA vs. t-PA alone in stroke. N Engl J Med 2015;372:2285-2295.

4. Jovin TG, Chamorro A, Cobo E, de Miquel MA, Molina CA, Rovira $A$, et al. Thrombectomy within 8 hours after symptom onset in ischemic stroke. N Engl J Med 2015;372:2296-2306.

5. Goyal M, Demchuk AM, Menon BK, Eesa M, Rempel JL, Thornton J, et al. Randomized assessment of rapid endovascular treatment of ischemic stroke. N Engl J Med 2015;372: 1019-1030.

6. Goyal M, Menon BK, van Zwam WH, Dippel DW, Mitchell PJ, Demchuk AM, et al. Endovascular thrombectomy after largevessel ischaemic stroke: a meta-analysis of individual patient data from five randomised trials. Lancet 2016;387:1723-1731.

7. Powers WJ, Derdeyn CP, Biller J, Coffey CS, Hoh BL, Jauch EC, et al. 2015 American Heart Association/American Stroke Association focused update of the 2013 guidelines for the early management of patients with acute ischemic stroke regarding endovascular treatment: a guideline for healthcare professionals from the American Heart Association/American Stroke Association. Stroke 2015;46:3020-3035.

8. Wahlgren N, Moreira T, Michel P, Steiner T, Jansen O, Cognard $C_{1}$ et al. Mechanical thrombectomy in acute ischemic stroke: consensus statement by ESO-Karolinska Stroke Update 2014/2015, supported by ESO, ESMINT, ESNR and EAN. Int J Stroke 2016;11:134-147.

9. Casaubon LK, Boulanger JM, Blacquiere D, Boucher S, Brown K, Goddard T, et al. Canadian stroke best practice recommendations: hyperacute stroke care guidelines, update 2015. Int J Stroke 2015;10:924-940.

10. Hong KS, Ko SB, Lee JS, Yu KH, Rha JH. Endovascular recanalization therapy in acute ischemic stroke: updated metaanalysis of randomized controlled trials. J Stroke 2015;17: 268-281.

11. Saver JL, Goyal M, van der Lugt A, Menon BK, Majoie $C B$, Dippel DW, et al. Time to treatment with endovascular thrombectomy and outcomes from ischemic stroke: a metaanalysis. JAMA 2016;316:1279-1288.

12. Lansberg MG, Straka M, Kemp S, Mlynash M, Wechsler LR, Jovin TG, et al. MRI profile and response to endovascular reperfusion after stroke (DEFUSE 2): a prospective cohort study. Lancet Neurol 2012;11:860-887.

13. Lansberg MG, Christensen S, Kemp S, Mlynash M, Mishra N,

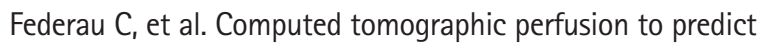
response to recanalization in ischemic stroke. Ann Neurol 2017;81:849-856.

14. Jovin TG, Liebeskind DS, Gupta R, Rymer M, Rai A, Zaidat 00, et al. Imaging-based endovascular therapy for acute ischemic stroke due to proximal intracranial anterior circulation occlusion treated beyond 8 hours from time last seen well: retrospective multicenter analysis of 237 consecutive patients. Stroke 2011;42:2206-2211.

15. Copen WA, Rezai Gharai L, Barak ER, Schwamm LH, Wu O, Kamalian $\mathrm{S}$, et al. Existence of the diffusion-perfusion mismatch within 24 hours after onset of acute stroke:dependence on proximal arterial occlusion. Radiology 2009;250:878-886.

16. Jovin TG, Saver JL, Ribo M, Pereira V, Furlan A, Bonafe A, et al. Diffusion-weighted imaging or computerized tomography perfusion assessment with clinical mismatch in the triage of wake up and late presenting strokes undergoing neurointervention with Trevo (DAWN) trial methods. Int J Stroke 2017; 12:641-652.

17. Albers GW, Lansberg MG, Kemp S, Tsai JP, Lavori P, Christensen $S$, et al. A multicenter randomized controlled trial of endovascular therapy following imaging evaluation for ischemic stroke (DEFUSE 3). Int J Stroke 2017;12:896-905.

18. Nogueira $R G$, Jadhav $A P$, Haussen $D C$, Bonafe $A$, Budzik RF, Bhuva $P$, et al. Thrombectomy 6 to 24 hours after stroke with a mismatch between deficit and infarct. N Engl J Med 2018; 378:11-21.

19. Albers GW, Marks MP, Kemp S, Christensen S, Tsai JP, OrtegaGutierrez S, et al. Thrombectomy for stroke at 6 to 16 hours with selection by perfusion imaging. N Engl J Med 2018;378: 708-718.

20. Powers WJ, Rabinstein AA, Ackerson T, Adeoye OM, Bambakidis NC, Becker K, et al. 2018 Guidelines for the early management of patients with acute ischemic stroke: a guideline for healthcare professionals from the American Heart Association/American Stroke Association. Stroke 2018;49:e46-e110.

21. Boulanger JM, Lindsay MP, Gubitz G, Smith EE, Stotts G, Foley N. Canadian stroke best practice recommendations for acute stroke management: prehospital, emergency department, and acute inpatient stroke care, 6th edition, update 2018. Int J Stroke 2018;13:949-984.

22. Australian Clinical Guidelines for Stroke Management. Chapter 8.2 neurointervention. https://app.Magicapp.Org/app\#/guideline/2638/section/32356. 2017. Accessed March 15, 2019.

23. United States Department of Health and Human Services. Acute Pain Management: Operative or Medical Procedures and Trauma. Rockville, MD: AHCPR, 1993;107.

24. Kudo K, Christensen $S$, Sasaki M, Østergaard L, Shirato $H$, Ogasawara K, et al. Accuracy and reliability assessment of CT and MR perfusion analysis software using a digital phantom. Radiology 2013;267:201-211.

25. Austein F, Riedel C, Kerby T, Meyne J, Binder A, Lindner T, et al. Comparison of perfusion CT software to predict the final in- 
farct volume after thrombectomy. Stroke 2016;47:2311-2317.

26. Kudo K, Sasaki M, Yamada K, Momoshima S, Utsunomiya $H_{\text {, }}$ Shirato $\mathrm{H}$, et al. Differences in CT perfusion maps generated by different commercial software: quantitative analysis by using identical source data of acute stroke patients. Radiology 2010;254:200-209.

27. Warach SJ, Luby M, Albers GW, Bammer R, Bivard A, Campbell $B C$, et al. Acute stroke imaging research roadmap III imaging selection and outcomes in acute stroke reperfusion clinical trials: consensus recommendations and further research priorities. Stroke 2016;47:1389-1398.

28. Brook RH, Chassin MR, Fink A, Solomon DH, Kosecoff J, Park RE. A method for the detailed assessment of the appropriateness of medical technologies. Int J Technol Assess Health Care 1986;2:53-63.

29. Wardlaw JM, Seymour J, Cairns J, Keir S, Lewis S, Sandercock P. Immediate computed tomography scanning of acute stroke is cost-effective and improves quality of life. Stroke 2004;35:2477-2483.
30. Kidwell CS, Chalela JA, Saver JL, Starkman S, Hill MD, Demchuk AM, et al. Comparison of MRI and CT for detection of acute intracerebral hemorrhage. JAMA 2004;292:1823-1830.

31. Brazzelli M, Sandercock PA, Chappell FM, Celani MG, Righetti $E_{1}$ Arestis $N$, et al. Magnetic resonance imaging versus computed tomography for detection of acute vascular lesions in patients presenting with stroke symptoms. Cochrane Database Syst Rev 2009;4:CD007424.

32. Jadhav AP, Desai SM, Kenmuir CL, Rocha M, Starr MT, Molyneaux $B J$, et al. Eligibility for endovascular trial enrollment in the 6- to 24-hour time window: analysis of a single comprehensive stroke center. Stroke 2018;49:1015-1017.

33. Hong KS, Bang OY, Kang DW, Yu KH, Bae HJ, Lee JS, et al. Stroke statistics in Korea: part I. Epidemiology and risk factors: a report from the Korean Stroke Society and clinical research center for stroke. J Stroke 2013;15:2-20.

34. Lee KJ, Kim BJ, Kim DE, Ryu WS, Han MK, Kim JT, et al. Nationwide estimation of eligibility for endovascular thrombectomy based on the DAWN trial. J Stroke 2018;20:277-279. 
Supplementary Table 1. List of responding participants

\begin{tabular}{|c|c|}
\hline Participants & Affiliation \\
\hline Jang-Hyun Baek & Department of Neurology, National Medical Center, Seoul, Korea \\
\hline Dong-Hyun Shim & Department of Neurology, Samsung Changwon Hospital, Sungkyunkwan University School of Medicine, Changwon, Korea \\
\hline Dong Geun Lee & Department of Neurology, Sejong General Hospital, Bucheon, Korea \\
\hline Hee-Kwon Park & Department of Neurology, Inha University Hospital, Incheon, Korea \\
\hline Woo-Keun Seo & Department of Neurology and Stroke Center, Samsung Medical Center, Sungkyunkwan University School of Medicine, Seoul, Korea \\
\hline Joonsang Yoo & Department of Neurology, Keimyung University Dongsan Medical Center, Daegu, Korea \\
\hline Jun Lee & Department of Neurology, Yeungnam University Hospital, Daegu, Korea \\
\hline Jin Soo Lee & Department of Neurology, Ajou University School of Medicine, Suwon, Korea \\
\hline Sam Yeol Ha & Department of Neurology, Inje University Haeundae Paik Hospital, Busan, Korea \\
\hline Jeong-Jin Park & Department of Neurology, Konkuk University Hospital, Seoul, Korea \\
\hline Si Baek Lee & Department of Neurology, Uijeongbu St. Mary's Hospital, College of Medicine, The Catholic University of Korea, Uijeongbu, Korea \\
\hline Jun Young Chang & Department of Neurology, Asan Medical Center, University of Ulsan College of Medicine, Seoul, Korea \\
\hline Ho Geol Woo & Department of Neurology, Soonchunhyang University Cheonan Hospital, Cheonan, Korea \\
\hline Sang Joon An & Department of Neurology, International St. Mary's Hospital, Catholic Kwandong University College of Medicine, Incheon, Korea \\
\hline Ji Hoe Heo & Department of Neurology, Severance Hospital, Yonsei University College of Medicine, Seoul, Korea \\
\hline Joung-Ho Rha & Department of Neurology, Inha University School of Medicine, Incheon, Korea \\
\hline Sun U. Kwon & Department of Neurology, Asan Medical Center, University of Ulsan College of Medicine, Seoul, Korea \\
\hline Keun-Sik Hong & Department of Neurology, Inje University Ilsan Paik Hospital, Goyang, Korea \\
\hline Sang-Bae Ko & Department of Neurology, Seoul National University Hospital, Seoul, Korea \\
\hline Hong-Kyun Park & Department of Neurology, Inje University Ilsan Paik Hospital, Goyang, Korea \\
\hline Jong S. Kim & Department of Neurology, Asan Medical Center, University of Ulsan College of Medicine, Seoul, Korea \\
\hline Byung-Chul Lee & Department of Neurology, Hallym University Sacred Heart Hospital, Anyang, Korea \\
\hline Joong Hyun Park & Department of Neurology, Inje University Sanggye Paik Hospital, Seoul, Korea \\
\hline Sang-Min Sung & Department of Neurology, Pusan National University Hospital, Busan, Korea \\
\hline Byoung-Soo Shin & Department of Neurology, Chonbuk National University Medical School, Jeonju, Korea \\
\hline Sungwook Yu & Department of Neurology, Korea University Anam Hospital, Seoul, Korea \\
\hline Tae-Jin Song & Department of Neurology, Ewha Womans University Mokdong Hospital, Seoul, Korea \\
\hline Byung Moon Kim & Department of Radiology, Severance Stroke Center, Severance Hospital, Yonsei University College of Medicine, Seoul, Korea \\
\hline Sang Hyun Suh & Department of Radiology, Gangnam Severance Hospital, Yonsei University College of Medicine, Seoul, Korea \\
\hline Cheolkyu Jung & Department of Radiology, Seoul National University Bundang Hospital, Seongnam, Korea \\
\hline Tae Gon Kim & Department of Neurosurgery, CHA Bundang Medical Center, CHA University, Seongnam, Korea \\
\hline Sang-Hoon Cha & Department of Radiology, Chungbuk National University College of Medicine, Cheongju, Korea \\
\hline Hyon Jo Kwon & Department of Neurosurgery, Chungnam National University Hospital, Daejeon, Korea \\
\hline Woong Yoon & Department of Radiology, Chonnam National University Hospital, Gwangju, Korea \\
\hline Hae Woong Jeong & Department of Radiology, Inje University Busan Paik Hospital, Busan, Korea \\
\hline Dong-Hun Kang & Department of Neurosurgery, School of Medicine, Kyungpook National University, Daegu, Korea \\
\hline Chang-Hyun Kim & Department of Neurosurgery, Keimyung University Dongsan Medical Center, Daegu, Korea \\
\hline Yoo Dong Won & Department of Radiology, Uijeongbu St. Mary's Hospital, College of Medicine, The Catholic University of Korea, Uijeongbu, Korea \\
\hline Jin Woo Kim & Department of Radiology, Inje University Ilsan Paik Hospital, Goyang, Korea \\
\hline Hong Jun Jeon & Department of Neurosurgery, Hallym University Kangdong Sacred Heart Hospital, Seoul, Korea \\
\hline Byungjoon Kim & Department of Radiology, Korea University Anam Hospital, Korea University College of Medicine, Seoul, Korea \\
\hline
\end{tabular}


Supplementary Table 2. Results of Delphi consensus

\begin{tabular}{|c|c|c|c|c|}
\hline Recommendations & $\begin{array}{c}\text { Delphi round } \\
\text { achieving consensus }\end{array}$ & $\begin{array}{c}\text { Agreement } \\
\text { (score 7-9) (\%) }\end{array}$ & $\begin{array}{c}\text { Uncertainty } \\
\text { (score 4-6) (\%) }\end{array}$ & $\begin{array}{l}\text { Disagreement } \\
\text { (score 1-3) }(\%)\end{array}$ \\
\hline \multicolumn{5}{|l|}{ Questions } \\
\hline $\begin{array}{l}\text { Is it necessary to update the recommendations of ERT between 6-24 hours } \\
\text { from last known normal time considering current clinical practice in Korea? }\end{array}$ & First round & 97.6 & 0.0 & 2.4 \\
\hline $\begin{array}{l}\text { Could multimodal imaging available on-site, other than the RAPID software } \\
\text { program, be used to select patients eligible for ERT between 6-24 hours? }\end{array}$ & First round & 82.9 & 9.8 & 7.3 \\
\hline \multicolumn{5}{|l|}{ ERT } \\
\hline $\begin{array}{l}\text { Recommendation 5: Option \# } 1 \\
\text { 5. In selected patients with acute ischemic stroke due to large vessel occlusion } \\
\text { in the anterior circulation presenting within } 6 \text { to } 24 \text { hours from last seen } \\
\text { normal, ERT is recommended when the patients meet the DAWN or DEFUSE } \\
3 \text { eligibility criteria (LOE Ib, GOR A) }\end{array}$ & First round & 29.3 & 31.7 & 36.6 \\
\hline $\begin{array}{l}\text { Recommendation 5: Option \#2 } \\
\text { 5. In selected patients with acute ischemic stroke due to large vessel occlusion } \\
\text { in the anterior circulation presenting within } 6 \text { to } 24 \text { hours from last seen } \\
\text { normal, ERT can be recommended when the patients have target mismatch } \\
\text { assessed by multimodal imaging and/or clinical deficit \& when reperfusion } \\
\text { by ERT is expected to improve the outcome of patients. For patient selection, } \\
\text { each institution is recommended to have its own criteria, which aids in the } \\
\text { timely and reasonable identification of patients with target mismatch in the } \\
\text { late time window (LOE III, GOR C). }\end{array}$ & First round & 80.5 & 17.1 & 2.4 \\
\hline $\begin{array}{l}\text { 6. In selected patients with acute ischemic stroke due to large vessel occlusion } \\
\text { in the posterior circulation presenting after } 6 \text { hours, ERT can be considered } \\
\text { for patients having favorable multimodal imaging profiles in consideration } \\
\text { of risks and benefits. Each center is encouraged to define its own patient se- } \\
\text { lection criteria (LOE IV, GOR C). }\end{array}$ & First round & 92.7 & 7.3 & 0.0 \\
\hline \multicolumn{5}{|l|}{ Neuroimaging evaluation } \\
\hline $\begin{array}{l}\text { 1. Non-contrast CT or MRI should be conducted to exclude hemorrhagic } \\
\text { stroke or other non-stroke etiologies (LOE III, GOR C). }\end{array}$ & First round & 95.1 & 4.9 & 0.0 \\
\hline $\begin{array}{l}\text { 2. Non-invasive vascular imaging (CT angiography or MR angiography) is rec- } \\
\text { ommended to confirm acute large artery occlusion for patients with major } \\
\text { ischemic stroke (LOE III, GOR C). }\end{array}$ & First round & 95.1 & 4.9 & 0.0 \\
\hline $\begin{array}{l}\text { 5. In selected patients who present within } 6 \text { to } 24 \text { hours from last seen nor- } \\
\text { mal, multimodal imaging for assessing collaterals, infarct core, or perfusion } \\
\text { (or clinical)-diffusion mismatch is recommended to select eligible patients } \\
\text { for ERT. Each center is encouraged to define its own imaging modality to } \\
\text { timely identify target mismatch (LOE III, GOR C). }\end{array}$ & First round & 85.4 & 4.9 & 7.3 \\
\hline
\end{tabular}

ERT, endovascular recanalization therapy; DAWN, Clinical Mismatch in the Triage of Wake Up and Late Presenting Strokes Undergoing Neurointervention with Trevo; DEFUSE 3, Endovascular Therapy Following Imaging Evaluation for Ischemic Stroke 3; LOE, level of evidence; GOR, grade of recommendation; CT, computed tomography; MRI, magnetic resonance imaging; MR, magnetic resonance. 
Supplemental Table 3. Comparison of changes in recommendations between previous and revised guidelines

\begin{tabular}{|c|c|c|}
\hline & Previous guideline in 2016 & Updated guideline in 2019 \\
\hline ERT & $\begin{array}{l}\text { 5. For patients with acute large artery occlusion in the anterior or } \\
\text { posterior circulation presenting after } 6 \text { hours, ERT can be consid- } \\
\text { ered for patients having favorable multimodal imaging profiles } \\
\text { regarding expected benefit and safety. Each center is encouraged } \\
\text { to define its own selection criteria (LOE IV, GOR C). }\end{array}$ & $\begin{array}{l}\text { 5. In selected patients with acute ischemic stroke due to large ves- } \\
\text { sel occlusion in the anterior circulation presenting within } 6 \text { to } 24 \\
\text { hours from last seen normal, ERT can be recommended when pa- } \\
\text { tients have target mismatch assessed by multimodal imaging and/ } \\
\text { or clinical deficit \& when reperfusion by ERT is expected to im- } \\
\text { prove the outcome of patients. For patient selection, each institu- } \\
\text { tion is recommended to have its own criteria, which can help in } \\
\text { the timely and reasonable identification of patients with target } \\
\text { mismatch in the late time window (LOE III, GOR C). }\end{array}$ \\
\hline \multirow[t]{3}{*}{$\begin{array}{c}\text { Neuroimaging } \\
\text { evaluation }\end{array}$} & $\begin{array}{l}\text { 1. Non-contrast CT or MRI should be conducted to exclude hemor- } \\
\text { rhagic stroke or other non-stroke etiologies (GPP). }\end{array}$ & $\begin{array}{l}\text { 1. Non-contrast CT or MRI should be conducted to exclude hemor- } \\
\text { rhagic stroke or other non-stroke etiologies (LOE III, GOR C). }\end{array}$ \\
\hline & $\begin{array}{l}\text { 2. Non-invasive vascular imaging (CT angiography or MR angiogra- } \\
\text { phy) is recommended to confirm acute large artery occlusion for } \\
\text { patients with major ischemic stroke (GPP). }\end{array}$ & $\begin{array}{l}\text { 2. Non-invasive vascular imaging (CT angiography or MR angiogra- } \\
\text { phy) is recommended to confirm acute large artery occlusion for } \\
\text { patients with major ischemic stroke (LOE III, GOR C) }\end{array}$ \\
\hline & & $\begin{array}{l}\text { 5. In selected patients who present within } 6 \text { to } 24 \text { hours from last } \\
\text { seen normal, multimodal imaging for assessing collaterals, infarct } \\
\text { core, or perfusion (or clinical)-diffusion mismatch is recommend- } \\
\text { ed to select eligible patients for ERT. Each center is encouraged to } \\
\text { define its own imaging modality to timely identify target mis- } \\
\text { match (LOE III, GOR C). }\end{array}$ \\
\hline
\end{tabular}

ERT, endovascular recanalization therapy; LOE, level of evidence; GOR, grade of recommendation; $C T$, computed tomography; MRI, magnetic resonance imaging; GPP, good practice point; MR, magnetic resonance. 